\title{
ANALYSIS OF HEMT TIME-EVOLUTION CHARACTERISTICS
}

\author{
Anthony E. Parker ${ }^{\dagger}$ and James G. Rathmell ${ }^{\ddagger}$ \\ $\dagger$ Macquarie University, Sydney AUSTRALIA 2109, tonyp@ieee.org \\ $\ddagger$ The University of Sydney, AUSTRALIA 2006, jimr@ee.usyd.edu.au
}

\begin{abstract}
A novel transient measurement of the time-evolution of drain characteristics is analyzed to separate thermal and trapping dispersions. The procedure extracts isothermal characteristics from the measured data and parameterizes thermal dispersion. A significant dispersion effect in the isothermal characteristics is then linked to leakage currents that are related to drain potential. The effect of impact ionization is also evident in the data. This analysis provides information necessary to formulate bias- and rate-dependent models of HEMTs.
\end{abstract}

\section{INTRODUCTION}

The variation of HEMT characteristics with operating condition is a serious problem for many applications in which the operating point depends on the signal conditions. For example, particular forms of asymmetric distortion and various modulation schemes give rise to changing average or dc conditions of an amplifier. To simulate these circuits, the operating-condition dependency must be measured, quantified and incorporated in HEMT models.

A strategy is presented here to measure and describe the dynamics of operating-condition dependency. It is based on measurement of the evolution in time of transistor characteristic curves, from pulsed-I(V) curves to steady-state dc curves. This shows the rich dynamics of the dispersion effects, Parker et al. (1), responsible for operating-condition dependency in HEMTs. This novel technique for analyzing the dynamics gives a much better perspective for understanding the phenomena.

\section{TIME-EVOLUTION CHARACTERISTICS}

A time-evolution measurement gives, for an initial operating condition, a set of $\mathrm{I}(\mathrm{V})$ characteristic curves as a function of time. An example for a HEMT is shown in Fig 1(a). An arbitrary-pulse semiconductor parameter analyzer (APSPA), Parker (1), Scott et al. (2), CNERF (3), was used for the measurement. The instrument has been enhanced to provided the stability and accuracy required for measurements over many decades of timefrom under $100 \mathrm{~ns}$ to over $100 \mathrm{~s}$. Figure 1(a) shows the responses to a step change in terminal potentials from an initial operating condition. Each surface is the drain current versus time and drain potential, for a constant gate potential. The front edge, at $100 \mathrm{~ns}$ after the step change, is the characteristic given by a fast pulsed-I(V) measurement. The rear edge, at $100 \mathrm{~ms}$, is the characteristic given by a dc measurement.

Each point in the characteristics was measured in isolation. That is, for each point, a step change from an initial operating condition was applied and the current was sampled over time. In order to determine timeevolution data at constant terminal potential, several points in the vicinity of each desired data point of the characteristic curve were measured and the current determined by software interpolation. Transconductance and drain conductance, used in the following analysis, were also numerically determined at each point. This was repeated for each point in the final characteristics. The data was efficiently gathered by the APSPA system, which was configured to sample current versus time while delivering wide pulses.

Figure 1(b) shows the conventional display of six sets of characteristics extracted from time-slices in Fig. 1(a). There are obvious changes due to thermal and kink effects and the time of occurrence of these is also evident. It is clear that it is impossible to interpret conventional characteristics without time information.

\section{THERMAL NORMALIZATION}

At high drain potentials and currents, the dominate cause of variation in current with time, observed in Figs 1(a) and 1(b), is heating due to power dissipation. Heating causes a reduction in drain current at high power points and is responsible for the apparent negative conductance in the dc characteristics. 
A simple thermal model describes the drain current as

$$
i_{D}(t)=i_{D\left(T_{o}\right)}[1-\delta \bar{P}(t)]
$$

where $i_{D\left(T_{o}\right)}$ is the isothermal current at time $t$ that would flow if there was no temperature-related variation due to the cumulative power dissipation $\bar{P}(t)$. The term $\delta$ is the thermal resistance-temperature coefficient parameter $\left[\mathrm{W}^{-1}\right]$, Parker (1), Parker and Root (4).

During the time-evolution of drain current, from its value at the beginning of a step change to its value after settling to a new operating point, the cumulative power dissipation can be described fundamentally by

$$
\bar{P}(t)=V_{Q} I_{Q}[1-q(t)]+\int_{0}^{t} v_{D} i_{D}(t-\tau) \frac{d q(\tau)}{d \tau} d \tau,
$$

where $V_{Q} I_{Q}$ is the quiescent power dissipation prior to the step change, $v_{D}$ is the drain potential stepped to at time $t=0$, and $q(t)$ is a normalized step response. Since it is not possible to explicitly isolate $q(t)$ from this expression, the following expression was used for this work:

$$
\bar{P}(t)=V_{Q} I_{Q}\left[1-q^{\prime}(t)\right]+q^{\prime}(t) v_{D} i_{D}(t),
$$

where $q^{\prime}(t)$ is an approximate step response that incorporates the differences between Eqs 2 and 3 . At the beginning of a pulse, the average power dissipation is that of the initial operating point, so $q(t)=0$ and $\bar{P}=$ $I_{Q} V_{Q}$. For the settled dc values $q^{\prime}(t)=1$, since each point becomes its own bias and thus $\bar{P}=i_{D} v_{D}=I_{D} v_{D}$ where $I_{D}=i_{D}(\infty)$ is the drain current value settled upon long after the step change. The step response $q(t)$ can be isolated from Eqs 1 and 3 as:

$$
q^{\prime}(t)=1-\left.\frac{1}{\delta\left[v_{D} i_{D}(t)-V_{Q} I_{Q}\right]}\left[\frac{i_{D}(t)}{I_{D}}-1\right]\right|_{v_{D} i_{D} \text { large, }}
$$

where $v_{D}$ and $i_{D}$ are specified as large because the thermal effect must dominate other dispersion effects in order to use this expression to extract $q^{\prime}(t)$ from measured data. This is true for data at high-power points and at times greater than $100 \mathrm{~ns}$.

The isothermal characteristics can be determined from measured data using Eqns (1) and (3), and $q(t)$ extracted from current versus time data at a high-power point. The isothermal characteristics are obtained by selecting a $\delta$ such that the currents at high-power points are constant in time. In this example $\delta=0.95[\mathrm{~mm} / \mathrm{W}]$, which gives the result shown in Fig. 1(c). This value of $\delta$ gives $q^{\prime}=0.05$ for the pulse samples at $100 \mathrm{~ns}$, which is consistent with there being some initial change in junction temperature prior to this sample.

At high drain potentials, the currents in the extracted isothermal characteristics do converge near a common value for each gate potential. Moreover, data from several initial conditions, not shown here, also converge to the same common values. This confirms that dispersion in this high-drain-potential region is dominated by self-heating and is well described by Eq. (1). That is, a single value for $\delta$ accounts for the time-dependence of drain current at high drain potentials and all initial conditions. This also implies that any non-thermal dispersion effects are not observed in this region of data. Note, however, that there are other effects present but they occur before the first sample at $100 \mathrm{~ns}$.

\section{TRAP-RELATED DISPERSION}

The isothermal characteristics exhibit significant non-thermal dispersion effects, which are often attributed to trapping phenomena. With this assumption, these can be described by a trap-related potential, $r(t)$, that, along with the gate potential, $v_{G}$, contributes to the overall channel-controlling potential $v_{C}=v_{G}+v_{T}$.

The change in isothermal current relative to the isothermal dc value, $I_{D\left(T_{o}\right)}$, is related to the change in traprelated potential relative to its dc value, $V_{T}$. This relation is approximately given by

$$
\Delta i_{D\left(T_{o}\right)} \approx \Delta v_{T} \cdot \frac{d i_{D\left(T_{o}\right)}}{d v_{G}}, \quad \text { with } \quad \frac{d i_{D\left(T_{o}\right)}}{d v_{G}}=\frac{d i_{D}(t)}{d v_{G}} \cdot\left[\frac{1}{1-\delta \bar{P}(t)}\right]^{2},
$$


where $\Delta i_{D\left(T_{o}\right)}=i_{D\left(T_{o}\right)}-I_{D\left(T_{o}\right)}, \Delta v_{T}=v_{T}-V_{T}$, and $\frac{d i_{D}(t)}{d v_{G}}$ is the transconductance determined numerically from the measured data.

Fig. 2(a) shows the time-evolution of the channel-controlling potential, $\imath_{C}$, with respect to $V_{T}$, for the data points shown in Fig. 1(a). Figure 2(b) shows six time-slices of $\Delta v_{T}$ for $v_{G}=0$ - the top surface in the 3-d graph of Fig. 2(a).

The change in trap-related potential can be described by a change in charge accumulated in traps. If this is true, then the traps are occupied at a rate dependent on the terminal potentials and at a rate proportional to the channel current. This is supported by the distinctive dependence on $v_{D}$ of the time constant for the change in $\Delta v_{T}$ that is observed in the data.

At potentials near or above that of the kink effect in the dc characteristics, there is a rapid, faster than $10 \mu \mathrm{s}$, increase in trap potential. This is consistent with trapping of charge related to the impact ionization that causes the kink, Somerville et al. (5) Webster et al. (6). At potentials below that of the kink, where impact ionization is negligible, Somerville (5), there is still significant dispersion with long time constants from $100 \mu$ s to over $100 \mathrm{~ms}$. These time constants are closely proportional to $\exp \left(-v_{D} / V_{B}\right)$, where $V_{B}$ is a positive constant. The probability of trap occupancy, and hence these time constants, is proportional to related currents. This suggests that the occupancy of traps affecting this dispersion is related to the drain-gate or drain-source leakage currents because they are also proportional to $\exp \left(-\left(v_{D}-v_{G}\right) / V_{B}\right)$ and $\exp \left(-v_{D} / V_{B}\right)$ respectively.

Figure 2(c), which shows $\Delta v_{T}$ at $1 \mu \mathrm{s}$ for the four values of $v_{G}$, indicates that the achievable trap-related potential is dependent upon the terminal potentials. The change in trap-related potential is much less near pinch-off than at high $v_{G}$. The hump in the controlling potential surfaces of Fig. 2 also indicates dependence on $v_{D}$.

\section{CONCLUSION}

Investigations have been carried out over several initial bias conditions - only one has been shown here. These show the variation of the trap-related potential with bias. This potential is seen to decrease or increase with time, depending upon the state that is established by the bias.

The analysis above has separated thermal and trap-related dispersion effects so that they can be incorporated in a simulation model. The thermal dispersion is modelled with established techniques, Parker and Skellern (7). A trap-related potential can be calculated at an internal node, which is controlled by impact ionization and leakage currents. Thus a bias- and rate-dependent model can be formulated with the results of this analysis.

\section{ACKNOWLEDGEMENT}

This work has been supported by the Australian Research Council, the CSIRO Division of Telecommunications and Industrial Physics, and Agilent Technologies.

\section{REFERENCES}

[1] A. E. Parker, J. Rathmell, and J. Scott, 2000, "Pulsed measurements," in The Modern Microwave and RF Handbook (M. Golio, ed.), ch. 4.1, pp. 4-68 - 4-95, USA: CRC Press.

[2] J. B. Scott, J. G. Rathmell, A. E. Parker, and M. M. Sayed, 1996, "Pulsed device measurements and applications," IEEE Trans. Microwave Theory and Techniques, vol. 44, pp. 2718-2723.

[3] CNERF, "Pulsed-bias semiconductor parameter analyser," 1999, http://www.elec.mq.edu.au/cnerf.

[4] A. E. Parker and D. E. Root, 1998, "Pulse measurements quantify dispersion in PHEMTs," in URSI Symposium on Signals, Systems, and Electronics, pp. 444-449, 29 Sept.- 2 Oct.

[5] M. H. Somerville, A. Ernst, and J. A. del Alamo, 2000, "A physical model for the kink effect in InAlAs/InGaAs HEMT's," IEEE Trans. Electron Devices, vol. 47, pp. 922-93.

[6] R. T. Webster, S. Wu, and A. F. M. Anwar, 2000, "Impact ionization in InAlAs/InGaAs/InAlAs HEMT's," IEEE Electron Device Letters, vol. 21, pp. 193-195. 
[7] A. E. Parker and D. J. Skellern, 1997, "A realistic large-signal MESFET model for SPICE," IEEE Transactions on Microwave Theory and Techniques, vol. 45, pp. 1563-1571.

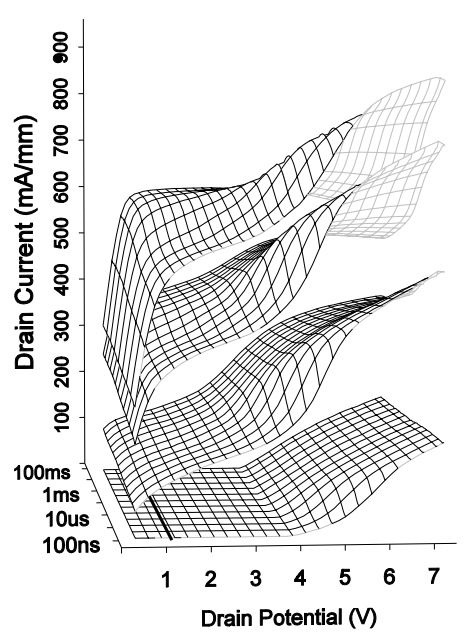

(a) Time-evolution characteristics.

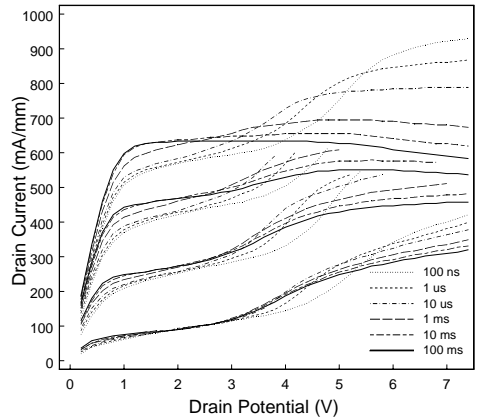

(b) Six time slices of the timeevolution characteristics.

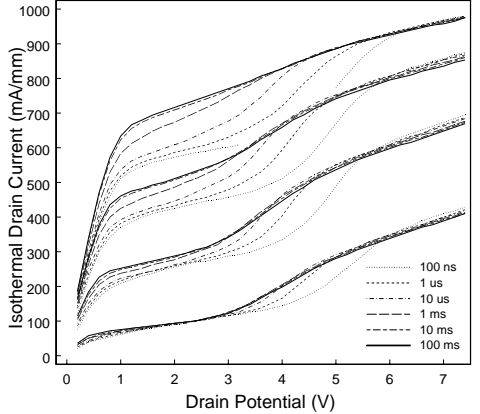

(c) Iso-thermal current extracted from the same six time slices.

Figure 1: Measured HEMT characteristics from the initial condition $V_{G}=-2.0 \mathrm{~V}, V_{D}=1.2 \mathrm{~V}$, with $v_{G}$ at $-1.2,-0.8,-0.4$, and $0.0 \mathrm{~V}$ as the parameter.

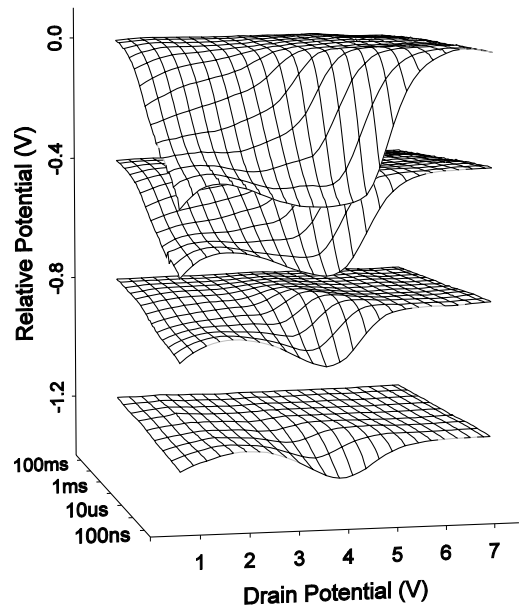

(a) Time-evolution of $v_{C}-V_{T}$, with $v_{G}$ at $-1.2,-0.8,-0.4$, and $0.0 \mathrm{~V}$.

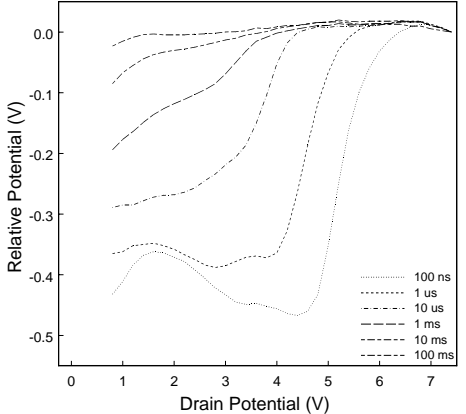

(b) Six time-slices of the change in trap-related potential, $\Delta v_{T}$, at $v_{G}=0$.

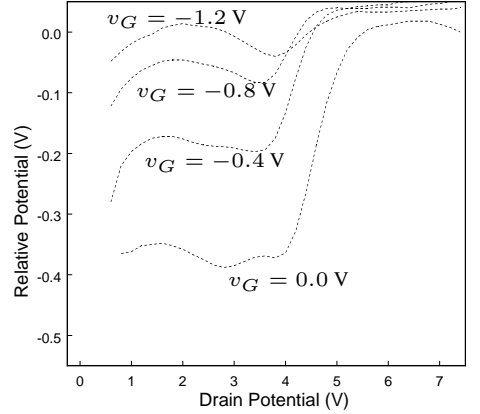

(c) Change in trap-related potential, $\Delta v_{T}$, at $1 \mu \mathrm{s}$ with $v_{G}$ at $-1.2,-0.8$, -0.4 , and $0.0 \mathrm{~V}$ as the parameter.

Figure 2: Effective channel-controlling potential derived from the HEMT characteristics for the initial condition $V_{G}=-2.0 \mathrm{~V}, V_{D}=1.2 \mathrm{~V}$ shown in Fig. 1. 